\title{
Fate of the organophosphate insecticide, chlorpyrifos, in leaves, soil, and air following application
}

\author{
Supta Das ${ }^{\dagger}$, Kimberly J. Hageman ${ }^{\S *}$, Madeleine Taylor ${ }^{\dagger}$, \\ Sue Michelsen-Heath ${ }^{\ddagger}$, Ian Stewart ${ }^{\dagger}$ \\ 'Department of Chemistry, University of Otago, Dunedin, New Zealand \\ ${ }^{\S}$ Department of Chemistry and Biochemistry, Utah State University, Utah, United States \\ Department of Zoology, University of Otago, Dunedin, New Zealand \\ *Corresponding Author \\ E-mail: kim.hageman@usu.edu; \\ Telephone: 1-435-797-0114
}

For Submission to Chemosphere

\section{ABSTRACT}

A field study was conducted to further our understanding about the fate and transport of the organophosphate insecticide, chlorpyrifos, and its degradation product, chlorpyrifos oxon. Leaf, soil and air sampling was conducted for 21 days after chlorpyrifos application to a field of purple tansy (Phacelia tanacetifolia). Air samples were collected using a high-volume air sampler (HVAS) and seven battery-operated medium-volume active air samplers placed around the field and on a $0.5-\mathrm{km}$ transect extending away from the field. Chlorpyrifos was detected every day of the sampling period in all matrices, with concentrations decreasing rapidly after application. Chlorpyrifos oxon was only detected in air samples collected with the HVAS during the first three days after application. Wind direction played a significant role in controlling the measured air concentrations in near-field samples. The SCREEN3 model and chlorpyrifos' Characteristic Travel Distance (CTD) were used to predict modeled chlorpyrifos concentrations in air along the transect. The concentration trend predicted by the SCREEN3 model was similar to that of measured concentrations whereas CTD-modelled concentrations decreased at a significantly 
31 slower rate, indicating that downwind chlorpyrifos concentrations in air were primarily controlled

32 by air dispersion. The SCREEN3-predicted chlorpyrifos concentrations were $\sim 5$ times higher than

33 measured concentrations, indicating that simple approaches for calculating accurate pesticide

34 fluxes are still needed. Finally, we found that measured concentrations in air on Days 0-2 at

35 locations up to $0.5-\mathrm{km}$ from the field were at levels considered concerning for human health.

\section{1. INTRODUCTION}

Pesticides applied to agricultural fields are subject to a number of fate processes including

39 degradation, volatilization followed by off-site vapor drift, accumulation in soil or plants, and

40 transport to surface or groundwater (Sarmah et al. 2004, Gao et al. 2012). Some of these processes

41 lead to pesticide exposure for non-target organisms, including humans, and therefore pesticide

42 concentrations are monitored and regulated in soil and water in most parts of the world, and in air

43 but to a lesser extent (Li and Jennings 2017). The relative contribution of each process to pesticide

44 fate depends on the physicochemical properties of the pesticide and other components of the

45 formulation, properties of the soil and crop, and meteorological conditions (temperature, wind

46 speed, relative humidity, and light intensity). The rates at which these processes occur are needed

47 to determine how long pesticides are effective against pests and potentially harmful to humans and

48 beneficial non-target organisms.

49 The environmental fate of pesticides has been the focus of many studies (e.g. Gao et al.

502012 and references). Herein, we focus on the semi-volatile organophosphate insecticide, 51 chlorpyrifos $(O, O$-diethyl $O-3,5,6$-trichloropyridin-2-yl phosphorothioate, CAS No. 5598-15-2).

52 Chlorpyrifos is one of the most frequently used insecticides in the world (Testai et al. 2010) but 53 requires careful management due to a variety of demonstrated effects on non-target organisms 
54 (John and Shaike 2015), including pollinators (Sanchez-Bayo and Goka 2014). Multiple studies

55 have also shown that human prenatal exposure to chlorpyrifos can result in the development of

56 autism, low birth-weight, attention deficit problems, and other developmental disorders (Perera et

57 al. 2005, Rauh et al. 2011, Silver et al. 2017). Chlorpyrifos breaks down in the environment to

58 chlorpyrifos oxon, which can be 10-1000 times more toxic than chlorpyrifos itself and can cause

59 acute cholinergic neurotoxicity in organisms (Flaskos 2012, Armstrong et al. 2013). Due to these

60 concerns, current chlorpyrifos regulations face increasing scrutiny (Mie et al. 2017, Centner 2018)

61 and it has been banned in the US state of California (California Environmental Protection Agency

62 2019) and several countries (Pesticide Action Network International 2019).

63 Several studies have investigated chlorpyrifos behavior in agricultural fields after

64 application. For example, Ngan et al. 2005 reported chlorpyrifos loss rates from soil following

65 application. Antonious et al. 2017 reported chlorpyrifos and chlorpyrifos oxon loss rates from

66 collard and kale foliage following application. Leistra et al. 2006 used micrometeorological

67 methods to calculate chlorpyrifos volatilization rates from a potato field. Zivan et al. 2016 used

68 measured concentrations at $70 \mathrm{~m}$ from a persimmon orchard and the pollutant dispersion model,

69 CALPUFF, to estimate concentrations in air surrounding the orchard; however, their work led

70 them to conclude that there is 'an urgent need for more measurements and modeling of atmospheric

71 transport of pesticides to rural communities' and that 'estimations of post-application rates are still

72 limited'. We also note that chlorpyrifos loss rates in soil, plants, and air have not been

73 simultaneously measured in any of the previously mentioned studies so a comparison of loss rates

74 from various media under identical conditions has not yet been possible. In addition, most studies

75 have monitored chlorpyrifos concentrations post-application for 3-7 days and therefore more

76 information is needed about its longer-term fate in an agricultural field. 
The objective of this study was to advance understanding about chlorpyrifos behavior in

78 agricultural environments by conducting a comprehensive investigation into its fate and loss rates

79 post-application. Chlorpyrifos was applied to a field of purple tansy (Phacelia tanacetifolia) as

80 part of a larger study on its effects on honey bees. Following its application, we measured

81 chlorpyrifos and chlorpyrifos oxon concentrations in soil, leaves, and air for 21 days. Air samples

82 were collected throughout the study period at seven locations around the field and along a transect

83 extending $500 \mathrm{~m}$ from the field. To investigate the relative importance of dispersion versus

84 depositional processes in controlling chlorpyrifos concentrations in air downwind of the field, we

85 compared the measured concentrations of chlorpyrifos in air along the transect to those predicted

86 using an air dispersion screening model, SCREEN3 (Lakes Environmental 2019, U.S.

87 Environmental Protection Agency 2019) and using chlorpyrifos' Characteristic Travel Distance

88 (CTD) (Bennett et al. 1998, Beyer and Matthies 2002). These two models were selected for

89 comparison because SCREEN3 predicts dispersion for any non-reacting chemical or atmospheric

90 particle whereas the CTD incorporates the physicochemical properties that affect a semi-volatile

91 chemical's atmospheric fate. We also evaluated various methods for predicting chlorpyrifos

92 volatilization flux, which is a key input parameter in SCREEN3, and compared measured

93 concentrations in air to human health standards.

94

95 2. METHODS

96 2.1 Sampling Site, Pesticide Application, and Sampling Approach.

97 The experimental field (Figure 1 and Supplemental Information (SI) Figure S1) was located

98 on a privately-owned farm in the Ida Valley, Central Otago, New Zealand (45² $12^{\prime} 59.46^{\prime \prime} \mathrm{S}$;

$\left.99169^{\circ} 42^{\prime} 6.56^{\prime \prime} \mathrm{E}\right)$. The total area of the field was 1.26 ha and it was mostly surrounded by rocks 


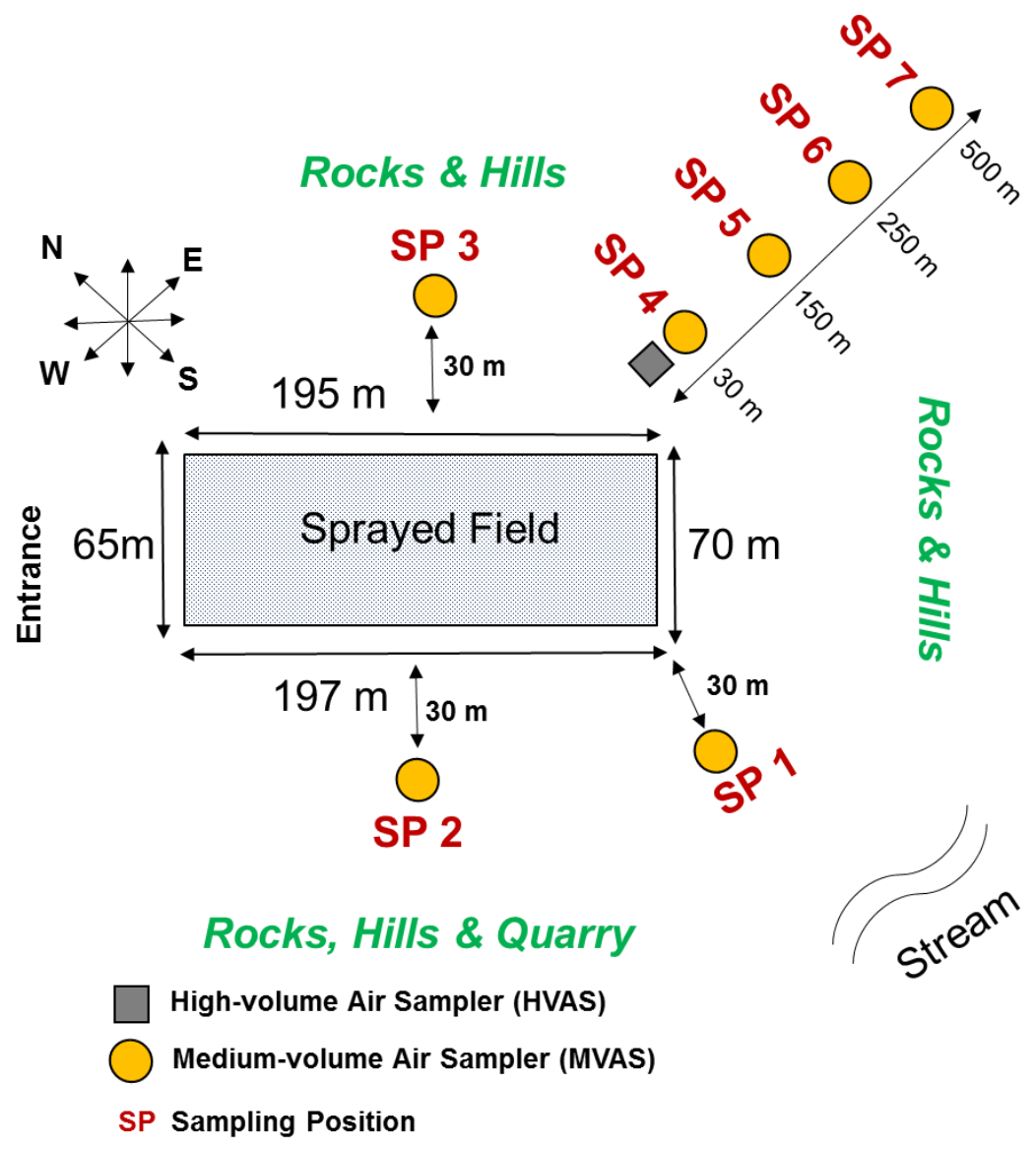

Figure 1. Experimental design indicating relative positions of sampling sites. In-field soil and leaf samples were collected at random locations within the sprayed field.

and small hills on three sides. Purple tansy (Phacelia tanacetifolia) seeds were sown on 21 October

101 2016. The field, with flowers in bloom, was sprayed with Lorsban ${ }^{\mathrm{TM}}$ 50EC (active ingredient:

102 chlorpyrifos) on 8 January 2017 (austral summer) starting at 8 am. The spray application was

103 carried out by a registered agrichemical contractor with a New Zealand Growsafe approved

104 Agrichemical Handler Certification. On the morning of the application, the tank mixture was

105 prepared according to label instructions (400 $\mathrm{mL}$ of Lorsban ${ }^{\mathrm{TM}} 50 \mathrm{EC}$ was added to $150 \mathrm{~L}$ of water)

106 and the mixture was applied to the field using a 24-m spray boom mounted on a truck.

107 Meteorological data were obtained from the nearest National Institute of Water and Atmospheric 
108 Research (NIWA) station (Lauder, $45^{0} 2^{\prime} 24.36^{\prime \prime} \mathrm{S} ; 169^{0} 41^{\prime} 3.084^{\prime \prime} \mathrm{E}$ ). The air temperature was

$10910^{\circ} \mathrm{C}$ and the wind speed was $0 \mathrm{~km} \mathrm{hr}^{-1}$ at the time of application.

110 Leaf and soil samples were collected from within the field. Air samples were collected at 111 several sampling positions (SPs) located $30 \mathrm{~m}$ outside of the field and along a transect extending $112500 \mathrm{~m}$ east of the field. After pesticide application, samples were collected for 21 days, using the

113 schedule shown in Table S1. A high-volume air sampler (HVAS) and seven battery-operated 114 medium-volume air samplers (MVASs) were used. The HVAS was used for three purposes: (a) its 115 relatively high sampling rate $\left(\sim 220 \mathrm{~L} \mathrm{~min}^{-1}\right)$ ensured that even low concentrations of chlorpyrifos 116 and its oxon would be detected, (b) it was used to calibrate the MVAS flow rate and (c) it could

117 be used to separately sample particle-bound and gas-phase chemicals. The MVAS sampling rates

118 were lower $\left(28 \mathrm{~L} \mathrm{~min}^{-1}\right)$ and could not separate particle-bound and gas-phase chemicals, but did 119 not require a power source so could be deployed at multiple locations around the experimental 120 field and along the transect.

\section{$122 \quad$ 2.2 Leaf and Soil Sampling.}

123 Leaf and soil samples were collected on each of the sampling days shown in Table S1. Purple

124 tansy leaves $(\sim 8 \mathrm{~g})$ were collected using gloved hands from randomly selected locations within the

125 sprayed field. Surface soil samples $(\sim 10 \mathrm{~cm}$ deep), which were collected from the same in-field 126 locations as leaves, were collected with a solvent-rinsed stainless steel sediment coring device. 127 Leaf samples were stored in baked aluminum foil packets inside plastic zip-lock bags and soil 128 samples were stored in pre-baked $\left(400{ }^{\circ} \mathrm{C}\right)$ amber glass jars. All samples were stored at $-20{ }^{\circ} \mathrm{C}$ and 129 analyzed within $\sim 120$ days of sample collection. 
131 in two representative samples collected on a dry day (Day 2) and a wet day (Day 8), respectively.

132 Total organic carbon, measured via the complete and instantaneous oxidation of the soil sample

133 by flash combustion using a Flash Smart Elemental Analyzer (Thermo Scientific, MA, USA), was

$1343.0 \%$ and $2.2 \%$ in the two samples, respectively.

\subsection{High-Volume Air Sampling.}

A high-volume air sampler (HVAS) (PUF 3300BRL/230, Hi-Q Environmental Products

138 Company, San Diego, CA) was deployed at SP 4, located $30 \mathrm{~m}$ from the eastern corner of the 139 experimental field (Figure 1 and Figure S2). The HVAS was operated with a diesel generator 140 because there was no power at the site. The sample cartridge head contained a 100-mm diameter 141 quartz fiber filter (QFF) (Munktell, New Zealand) to collect particle-bound chemicals and a glass 142 cartridge containing a polyurethane foam (PUF)/XAD-2 'sandwich' to collect gas-phase 143 chemicals. The PUF/XAD-2 sandwich contained 10 g XAD-2 resin (Restek, Australia) held 144 between a 3-inch (6-cm diameter, 7.6-cm length) and 1-inch (6-cm diameter, 2.5-cm length) PUF 145 plug (Restek, Australia). The HVAS was calibrated using a 10-cm adaptor plate (HI-Q 146 Environmental Products Company, CA, USA) and a digital manometer (Testo 511, Testo AG, 147 VIC, Australia). The mean flow rate was $220 \mathrm{~L} \mathrm{~min}^{-1}$. Prior to use, all QFF and glass cartridges 148 were baked for $4 \mathrm{~h}$ at $400{ }^{\circ} \mathrm{C}$. PUF plugs and XAD-2 were cleaned prior to deployment using 149 pressurized liquid extraction according to the method described in Section I of the SI and Table $150 \mathrm{~S} 2$.

151 At $\sim 8$ am on each of the sampling dates shown in Table S1, a QFF and PUF/XAD-2 cartridge 152 were installed in the HVAS. Following six hours of sampling, the sample cartridge and the QFF 
153 were removed, wrapped separately with pre-baked aluminum foil, and stored in a zip-lock bag in 154 an insulated container with ice blocks. After transporting samples to the laboratory, PUF and

155 XAD-2 were separated from the cartridge and stored separately in amber glass jars at $-20{ }^{\circ} \mathrm{C}$ until 156 analysis (within 120 days of sample collection). The 3- and 1-inch PUF plugs from the first three 157 sampling events were stored and extracted separately for breakthrough analysis. The 3- and 1-inch 158 PUF plugs from all other sampling events were stored and extracted together.

\subsection{Medium-Volume Air Sampling.}

161 The MVASs (SI Figure S3) were designed and built at the Department of Chemistry 162 Workshop, University of Otago. Each sampler body was made of stainless steel. The two chambers

163 inside the sampler were separated by a stainless steel plate with a 55-mm diameter hole in it. The 164 bottom chamber contained a 12-V fan (ebm-papst Inc., Australia) while the upper chamber held 165 the glass sampling cartridge. A glass sampling cartridge, containing a PUF/XAD-2 sandwich, was 166 positioned between the lower plate and a removable cover, with rubber seals on both ends. The

167 glass cartridges were identical to those used in the HVAS. A wind/rain shield was used to protect 168 the sampling cartridge without blocking air flow. A digital timer was used to control the sampling 169 time. Particles were not collected separately since the addition of QFF would have significantly 170 decreased the flow rate; therefore, both particle-bound and gas-phase chemicals were trapped in 171 the PUF/XAD-2 sampling cartridges.

172 MVASs were deployed at SPs 1-4 (Figure 1), which were located $30 \mathrm{~m}$ from the south corner, 173 the southwest border, the northeast border and the east corner of the experimental field, 174 respectively. Samples were not collected on the northwest border of the field due to the position 175 of the field entrance. An additional three MVASs were deployed at SPs 5-7 (Figure 1) and formed 
176 a transect extending $500 \mathrm{~m}$ to the east of the experimental field. The transect extended eastward

177 because we expected it to be the dominant downwind direction. The 3- and 1-inch PUF plugs from

178 the first three sampling events were stored and extracted separately for breakthrough analysis. At

$179 \sim 8$ am on each of the sampling days, a PUF/XAD-2 cartridge was installed in the MVAS. After

180 sampling for six hours, the sample cartridge was removed and stored in the same way as those

181 used with the HVAS.

182 The flow rate of the MVAS located at SP 4 was calculated for each sampling date using the

183 mass of chlorpyrifos measured in the MVAS cartridge ( $M_{\mathrm{CHL}}$, MVAS) and the chlorpyrifos

184 concentration in air determined from the co-located HVAS $\left(C_{\mathrm{CHL}}\right.$,air $)$ using equation 1 . The volume 185 of air sampled by the MVAS ( $V_{\text {air,MVAS }}$ ) was calculated with equation 1,

$$
V_{\text {air, } \operatorname{MVAS}}=M_{\mathrm{CHL}, \text { MVAS }} \mathrm{x} \frac{1}{C_{\mathrm{CHL}, \text { air }}}
$$

189 The mean MVAS flow rate, which was calculated by dividing $V_{\text {air,MVAS }}$ by the sampling time $(6 \mathrm{~h})$

190 for each of the nine sampling dates, was $28 \mathrm{~L} \mathrm{~min}^{-1}$.

\section{$192 \quad 2.5$ Chemicals.}

High-purity dichloromethane (>99.98\%), ethyl acetate $(>99.9 \%)$, hexane $(>98 \%)$, and

194 acetone (>99.98\%) were obtained from Merck (Germany). Chlorpyrifos was purchased from Fluka

195 (Germany) and chlorpyrifos oxon from Thermo Fisher Scientific (MA, USA). Chlorpyrifos-d10

196 was obtained from Cambridge Isotope Laboratories (MA, USA).

197

198 


\subsection{Analyte Extraction and Analysis.}

200 Chlorpyrifos and chlorpyrifos oxon were extracted from leaf, soil, and air sampling media

201 using pressurized liquid extraction with an Accelerated Solvent Extractor (ASE-350) from Thermo 202 Fisher Scientific (MA, USA). Chlorpyrifos was quantified with an Agilent 6890N gas 203 chromatograph coupled to an Agilent 5957 mass selective detector (GC-MS) (CA, USA). 204 Chlorpyrifos oxon was quantified with a Thermo Fisher Scientific TSQ Quantum Access MAX 205 Triple Quadruple Mass spectrometer (MA, USA). Detailed descriptions of the extraction and 206 instrumental procedures are provided in SI Sections II and III.

207 We did not target other potential chlorpyrifos degradation products, such as 3,5,6-trichloro208 2-methoxypyridinol and 3,5,6-trichloro-2-methoxypyridine, since previous studies have shown 209 that transformation to them is low relative to chlorpyrifos oxon (U.S. Environmental Protection 210 Agency 2018) and since they are not considered residues of concern due to relatively low toxicity 211 (U.S. Environmental Protection Agency 2011, Solomon et al. 2014).

\section{$213 \quad 2.7$ Quality Assurance.}

214 We quantified analytes in background samples, field blanks, and laboratory blanks. Method 215 recovery experiments for each sample matrix were conducted (Figure S4) and air sampling

216 methods were tested for breakthrough. Details regarding quality assurance can be found in SI 217 Section IV.

$219 \quad 2.8$ Modelling Atmospheric Pesticide Transport.

220 SCREEN3 is the screening version of the Gaussian plume Industrial Source Complex (ISC3) 221 model used by the US Environmental Protection Agency (EPA) (Lakes Environmental 2019, U.S. 
222 Environmental Protection Agency 2019). It is designed to estimate maximum pollutant

223 concentrations at defined distances from the pollutant source when the emission flux at the source

224 is known. All SCREEN3 input parameters are provided in SI Table S5. In all simulations, we used

225 the 'Area' option, a stability factor of 4 (default value), a wind speed of $5.9 \mathrm{~m} \mathrm{~s}^{-1}$ (the mean value

226 during our first sampling period, Table S5), and the angle describing the transect extending to the

227 east of our field (Figure 1). For the emission flux used in SCREEN3, we calculated a chlorpyrifos

228 volatilization flux from the field using our measured leaf concentration, as described in SI Section

229 V. We also tested several chlorpyrifos volatilization fluxes reported in the literature (Table S6),

230 and one calculated using the approach of Woodrow et al. 1997 for comparison.

231 The CTD approach was developed to predict the transport potential of semi-volatile

232 compounds in the atmosphere (Bennett et al. 1998, Beyer and Matthies 2002). CTD is the distance

233 from the source region at which the concentration of a chemical is reduced by $63 \%$. The ELPOS

234 model uses the chemical and physical properties of the chemical to predict CTD. The ELPOS input

235 parameters that we used are provided in Table S7. Equation 2 was used to generate ELPOS-

236 modeled chlorpyrifos concentrations in air at distances downwind from the field (Bennett et al.

237 1998).

$$
\left.C_{\mathrm{x}}=C_{0} \mathrm{e}^{(-x / \mathrm{CTD})} \quad \text { (Eq. } 2\right)
$$

239 where $C_{\mathrm{x}}$ is the concentration of pesticide in the air at distance $x$ and $C_{0}$ is the concentration at the 240 source, which is the agricultural field in our case. 


\section{3. RESULTS \& DISCUSSION}

\section{$246 \quad 3.1$ Weather Conditions.}

247 The pesticide application day was sunny with no rainfall, and the mean temperature during the 6-

$248 \mathrm{~h}$ sampling event was $18{ }^{\circ} \mathrm{C}\left(\right.$ Table S8). The highest $\left(27^{\circ} \mathrm{C}\right)$ and lowest $\left(13^{\circ} \mathrm{C}\right)$ mean 6-h sampling

249 period temperatures occurred on Days 2 and 12, respectively. The winds during sampling were

250 relatively calm on the first two days of the study (Figures S5 and S6). After that, the strongest

251 winds generally came from the northwest, with exceptions on Days 5 and 9 when the strongest

252 winds came from the west and southeast, respectively. Figure S6 shows that during the first week

253 after application, winds were calm in the morning and strong at night, with the strongest winds

254 mainly coming from the northwest.

255

$256 \quad 3.2$ In-field Chlorpyrifos Concentrations in Leaves.

257 The mean concentration of chlorpyrifos in the first leaf samples collected after application 258 (Day 0) was $21.6 \mu \mathrm{g} \mathrm{g}^{-1}$ (Figure 2 and Table S9). The concentration then dropped rapidly such that 259 on Day 1, it was $\sim 20 \%$ of the first measured concentration after application. Following this initial 260 rapid loss, the concentration remained relatively constant until the end of the study. The final 261 concentration of chlorpyrifos in leaves was $1.1 \mu \mathrm{g} \mathrm{g}^{-1}$ or $\sim 5 \%$ of the initial concentration after 262 application. The concentration trend was best described by a power curve, with the time for 263 dissipation to half of the initial concentration $\left(\mathrm{DT}_{50}\right)$ being $0.4 \mathrm{~h}$ (Table S10). Chlorpyrifos oxon 264 was not detected in leaves. 


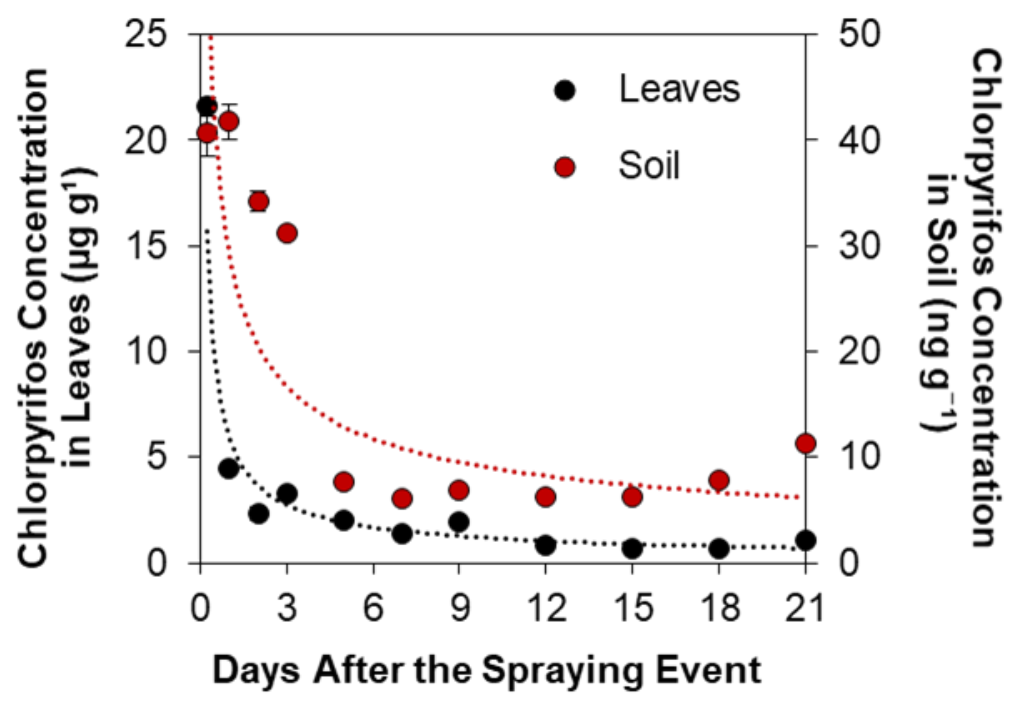

Figure 2. Chlorpyrifos concentrations in leaf and soil samples collected from within the field. Both fitted lines are power curves. Error bars, barely discernable in most cases, indicate \pm 1 standard deviation. All samples were analyzed in triplicate.

The range of $\mathrm{DT}_{50}$ values previously reported for chlorpyrifos dissipation from various leaf types is large ( 0.9 to $161 \mathrm{~h}$ ) (Table $\mathrm{S} 10)$. Interestingly, our $\mathrm{DT}_{50}$ value $(0.4 \mathrm{~h})$ was lower than any

267 of these. Pesticide dissipation rates from leaves are affected by volatilization, wash-off with 268 precipitation, and degradation. It did not rain during the first day of our study so wash-off was not

269 responsible for the particularly fast loss rate. While it is possible that photodegradation occurred,

270 the laboratory experiments conducted by Lester et al. 2017 showed that volatilization is the main

271 pathway for chlorpyrifos loss from lemon leaves. Pesticide concentrations on leaf surfaces may

272 decrease over time due to penetration into deeper layers; however, we measured the total 273 concentration in leaves and not just that on the surface.

274 The variability in $\mathrm{DT}_{50}$ values observed in Table S10 may be due to a number of factors, 275 including plant and field properties as well as meteorological conditions. Nonetheless, we note 276 that the mean air temperature was similar during our experiment $\left(18^{\circ} \mathrm{C}\right)$ and that by Leistra $e t a l$. $2772006\left(21^{\circ} \mathrm{C}\right)$, but the mean wind speed during our experiment $\left(5.9 \mathrm{~m} \mathrm{~s}^{-1}\right)$ was much higher than 
278 the range reported by Leistra et al. $2006\left(2.3\right.$ to $\left.3.5 \mathrm{~m} \mathrm{~s}^{-1}\right)$. Thus, wind speed likely contributed

279 significantly to the fast dissipation rate in our study. In any case, it is clear that chlorpyrifos DT 50

280 values from leaves are highly variable and that this research area could benefit from a more

281 systematic investigation into the factors that affect it. It is also worth noting that in some

282 experiments that report chlorpyrifos $\mathrm{DT}_{50}$ values from leaves, concentrations represent those on

283 leaf surfaces while in others (such as ours), they represent total concentrations. Significant

284 differences in surface versus total pesticide loss rates can be expected.

285 The chlorpyrifos concentration in leaves on Day 21 of our study was $>20$ times higher than

286 the maximum residue limits (MRLs) for leafy vegetables, herbs and edible flowers, which ranges

287 from $0.01-0.05 \mu \mathrm{g} \mathrm{g}^{-1}$ (European Food Safety Authority 2015). Although purple tansy is not a

288 harvestable crop and pesticide fate varies by plant species, this suggests chlorpyrifos residues in

289 edible leafy plants may be higher than expected and should be monitored carefully.

$291 \quad 3.3$ In-field Chlorpyrifos Concentrations in Soil.

292 The mean concentration of chlorpyrifos in the first soil samples collected after application

293 (Day 0) was $41 \mathrm{ng} \mathrm{g}^{-1}$ (Figure 2 and Table S9), which is 500 times lower than that measured in

294 leaves on Day 0. This indicates that leaves intercepted most of the chlorpyrifos during application.

295 The chlorpyrifos concentration decreased, but not as rapidly as it did from leaves, such that on Day

296 1, the concentration was $\sim 80 \%$ of the first measured concentration. From Day 5 to the end of the

297 study, the concentration was relatively stable and the concentration on the final day of the study

298 was $11.3 \mathrm{ng} \mathrm{g}^{-1}$, or $\sim 30 \%$ of the initial concentration. The concentration trend was best described

299 by a power curve, with the $\mathrm{DT}_{50}$ being $2 \mathrm{~h}$ (Table S10). Chlorpyrifos oxon was not detected in soil. 
In a review of the fate of chlorpyrifos in the environment, Mackay et. al. 2014 reported DT 50

301 values for chlorpyrifos in soil ranging from 168 to $720 \mathrm{~h}$ (7 to $30 \mathrm{~d}$ ) (Mackay et al. 2014). Thus,

302 our values and those reported by Ngan et al. 2005 are orders of magnitude lower than those

303 reported by Mackay et al. 2014 or those measured by Montemurro et al. 2002 in an orange grove

304 (Table S10). While our experiment and that of Ngan et al. 2005 were conducted with freshly

305 applied chlorpyrifos, the rates reported by Mackay et al. 2014 were likely determined for 'aged'

306 chlorpyrifos that had bound more tightly to soils over time. The orange grove studied by

307 Montemurro et al. 2002 may have also contained aged chlorpyrifos. There is evidence that

308 chlorpyrifos degrades faster in alkaline than acidic soils (Racke 1993); however, since our soils

309 were slightly acidic, this does not explain the relatively fast loss we observed.

310 The chlorpyrifos concentrations we measured in soil were $\sim 5$ times lower than the median

311 lethal dose for earth worms (210 $\left.\mathrm{ng} \mathrm{g}^{-1}\right)$ (Tomlin 2006), suggesting that ground-dwelling

312 organisms may be largely protected from high pesticide exposure when plants intercept a high

313 percentage of applied pesticides.

\section{3.4 Near-field Chlorpyrifos and Chlorpyrifos Oxon Concentrations in Air.}

316 The gas-phase chlorpyrifos concentrations reported in Figure 3 represent the total 317 concentration found in PUF and XAD-2 using the HVAS; however, very little chlorpyrifos was 318 found in XAD-2 (on average, the mass found in XAD-2 was $1.8 \%$ of that found in PUF; Table 319 S11). On most sampling days, the gas-phase concentrations were also much higher than the 320 particle-bound concentrations (Figure 3 and Table S11), which may simply be due to a low 321 concentration of particles in air at our site. 


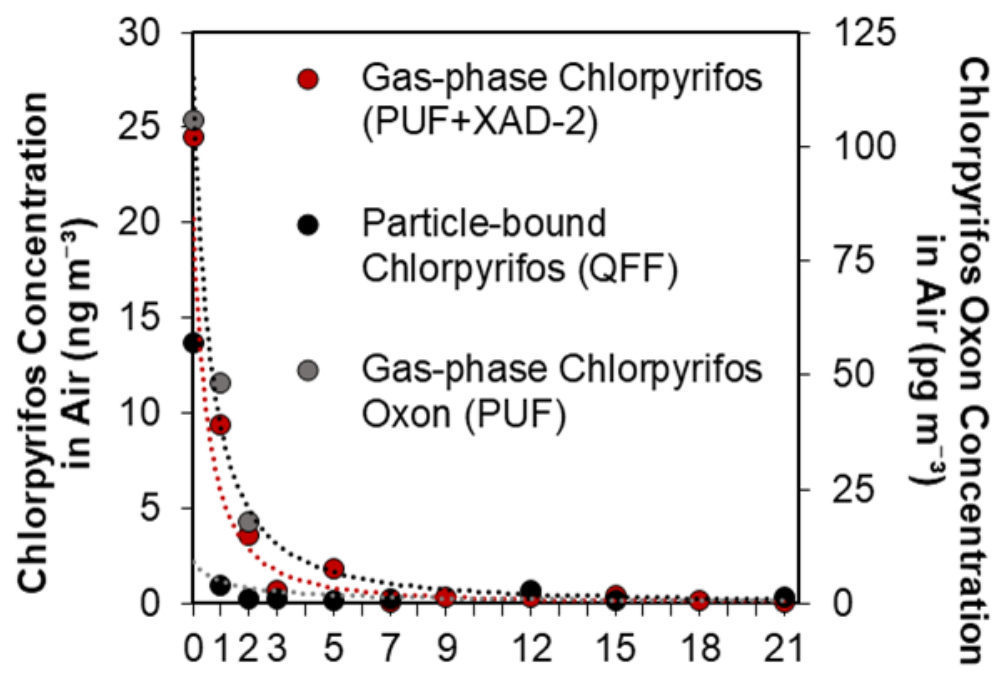

Days After the Spraying Event

Figure 3. Gas-phase chlorpyrifos, gas-phase chlorpyrifos oxon, and particle-bound chlorpyrifos concentrations measured with HVAS at sampling position 4. All three fitted lines are power curves. Error bars are not shown since air sampling was not conducted in triplicate.

The gas-phase and particle-bound concentrations at SP 4 in the first samples collected after

324 chlorpyrifos application (Day 0) were $\sim 24 \mathrm{ng} \mathrm{m}^{-3}$ and $\sim 13 \mathrm{ng} \mathrm{m}^{-3}$, respectively (Figure 3 and

325 Table S11). The concentrations in air decreased rapidly over the course of the study (Figure 3).

326 Again, concentration trends were best described by power curves; the DT 50 of the gas-phase and

327 particle-bound chlorpyrifos were 13 and $0.3 \mathrm{~h}$, respectively (Table S10). The particle-bound

328 concentrations may have decreased more rapidly than the gas-phase concentrations due to

329 particles generated during chlorpyrifos application quickly settling out. It is interesting that the

330 DT $_{50}$ for gas-phase chlorpyrifos was much higher than it was for leaves or soil. Also, our DT 50

331 values for gas-phase chlorpyrifos were 3-4 times higher than those reported by Guardino et al.

3321998 and Mackay et al. 2014. High variabilities in DT50 values for air from different studies are

333 not surprising since these values are highly dependent on meteorological conditions. 
A deviation from the smooth decreasing concentration trend was observed on Day 5 when

335 the gas-phase concentration was higher than expected. This increase was correlated with a switch

336 in wind direction; on Days 0-4, winds mainly came from the northeast and northwest but on Day

337 5, they came from the west, i.e. directly across the sprayed field towards SP 4 (Figure S5 and Table 338 S11).

339 The highest chlorpyrifos oxon concentration was $105.8 \mathrm{pg} \mathrm{m}^{-3}$ (Figure 3 and Table S11) and 340 was measured in the first HVAS-PUF sample collected after application (Day 0). Chlorpyrifos 341 oxon was detected in PUF but not in XAD-2. Zivan et al. 2016 also reported that chlorpyrifos oxon 342 was not present in the XAD-2 used in their high-volume air sampler (Zivan et al. 2016). Our HVAS 343 samples confirm the presence of chlorpyrifos oxon in air near the field until three days after the 344 spray event (Figure 3 and Table S11). However, chlorpyrifos oxon concentrations were very low 345 compared to chlorpyrifos concentrations. Interestingly, our $\mathrm{DT}_{50}$ for chlorpyrifos oxon was lower 346 than that for chlorpyrifos (Table S10) whereas Mackay et al. 2014 reported the opposite 347 relationship. Although chlorpyrifos oxon is more toxic than chlorpyrifos, our results support the 348 conclusion drawn by Mackay et. al. 2014 that chlorpyrifos oxon concentrations in air near sprayed 349 fields are not high enough to present a major concern. 


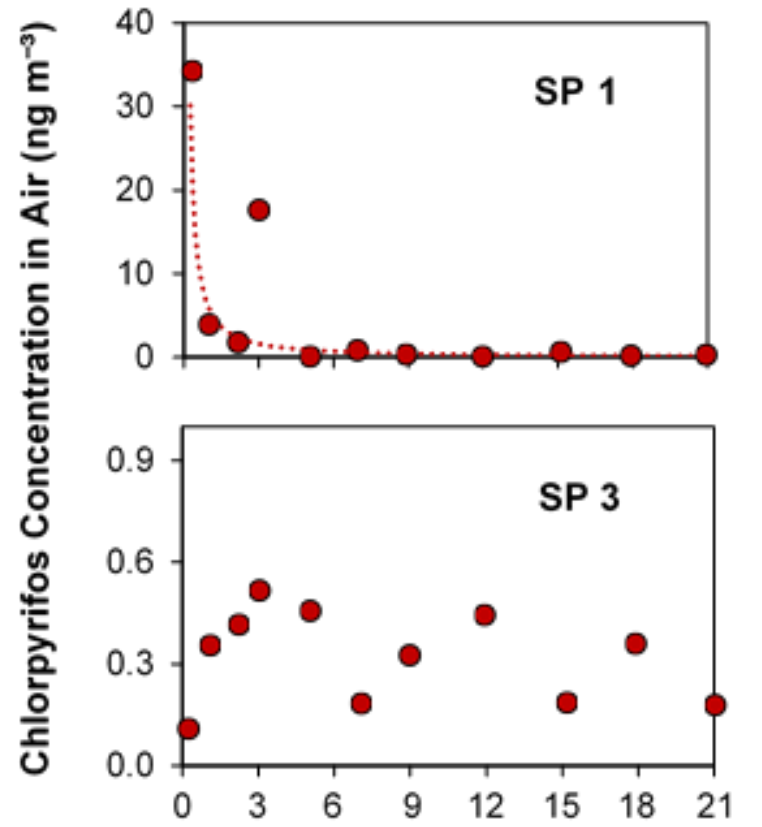

Days after Spraying


Days after Spraying

Figure 4. Chlorpyrifos concentrations measured with MVASs from sampling positions (SPs) 1-4. All three fitted lines are power curves. Error bars are not shown since air sampling was not conducted in triplicate.

The concentrations of chlorpyrifos at the various positions located at 30-m distances from

351 the field, as determined by the MVASs, are shown in Figure 4. The highest concentrations were

352 measured at SP $1\left(34 \mathrm{ng} \mathrm{m}^{-3}\right)$ and SP $2\left(28 \mathrm{ng} \mathrm{m}^{-3}\right)$ on Day 0 (Figure 4 and Table S12); these SPs

353 were on the southern border of the field (Figure 1). This is not surprising since the strongest winds

354 during the study came from the north (Figure S5). The concentrations in air decreased rapidly at

355 all SPs except SP 3. Throughout the study, the concentrations at SP 3, which was on the northeast

356 border of the field (Figure 1), were particularly low. This can be explained because no strong winds

357 came from the southwest direction during the study. The deviations from expected concentrations

358 observed at SP 1 on Day 3 and SP 4 on Day 5 can also be explained by shifts in wind direction on

359 those respective days (Figure S5). Our results show that the pesticide concentrations in air during 
360 the month following application can vary considerably on different sides of the sprayed field and

361 that these variations can generally be explained by wind direction.

363 3.5 Chlorpyrifos Concentrations in Air along the Transect.

Figure S7 and Table S12 show the chlorpyrifos concentrations in air along the transect

365 extending $500 \mathrm{~m}$ to the east of the sprayed field. Chlorpyrifos oxon was not detected in any of

366 these samples, presumably because of the lower sampling rate of the MVAS compared to that of

367 the HVAS. At SPs 4-6, the highest chlorpyrifos concentrations were measured in the first sample

368 collected after application (Day 0) and rapid decreases were observed after that (Figure S7). It is

369 interesting that this trend was not observed at SP 7, which was furthest from the experimental field.

370 Although the SP 4 data on Day 0 is not available due to a sampling problem, it appears from the

371 fitted curves that the concentration on Day 0 decreased along the transect from SP 4 to SP 7. An

372 interesting spike in concentration was observed at SP 7 on Day 3. This spike may be explained

373 because on the same day, the strongest winds were primarily blowing from the northwest (Figure

374 S5), across the sprayed field towards the sampler transect.

\subsection{Comparing Measured and Modeled Concentrations in Air along the Transect.}

377 In the SCREEN3 model, the emission flux at the source strongly influences modelled 378 chemical concentrations in air. For pesticide applications, the emission flux is equivalent to the 379 volatilization flux from the field. In Table S6, we compared the chlorpyrifos volatilization flux 380 calculated from our measured concentrations in leaves to previously reported rates determined 381 using micrometeorological methods (Leistra et al. 2006, Mackay et al. 2014), as well as the flux 382 estimated from vapor pressure using an empirical equation developed by Woodrow et al. 1997. 
383 The reported chlorpyrifos volatilization rates cover several orders of magnitude and our calculated

384 flux falls within this range. Since our approach is considerably simpler and cheaper than the ones

385 reliant on micrometeorological measurements, and more field-specific than the estimation

386 approach presented by Woodrow et al. 1997, it is worth further exploration as a viable alternative.

387 The SCREEN3-predicted chlorpyrifos concentrations obtained when using our calculated

388 flux were $\sim 5$ times higher than the measured concentrations (Table S13). The predicted 389 concentrations deviated further from measured values when using cited chlorpyrifos fluxes from

390 the literature (Table S13), demonstrating that pesticide volatilization fluxes are not transferable

391 between studies. The SCREEN3-predicted concentrations obtained when using the volatilization

392 flux predicted using the empirical equation derived by Woodrow et al. 1997 (Table S6) were also

393 much higher than our measured values (data not shown). Many factors related to the crop, field

394 conditions, application protocol, and meteorology could affect the volatilization flux; most

395 importantly, these results highlight the importance of volatilization flux on downwind 396 concentrations and the need for accurate methods for predicting it.

397 To determine the effect of air dispersion on the chlorpyrifos concentrations we measured 398 along the transect, we focused on the concentration trend produced by SCREEN3. This trend is 399 affected by the air stability factor, but not by wind speed, field size, or the emission flux. To 400 determine if the chlorpyrifos concentration trends we measured along our transect on Days 0,1 , 401 and 2 were controlled primarily by air dispersion, we normalized the modelled concentrations so 402 they matched those measured close to the field edge (Figure 5). These plots show that on all three 403 days, the measured and SCREEN3-modelled concentration trends along the transect were very 404 similar, indicating that the concentration trends were primarily controlled by air dispersion. 


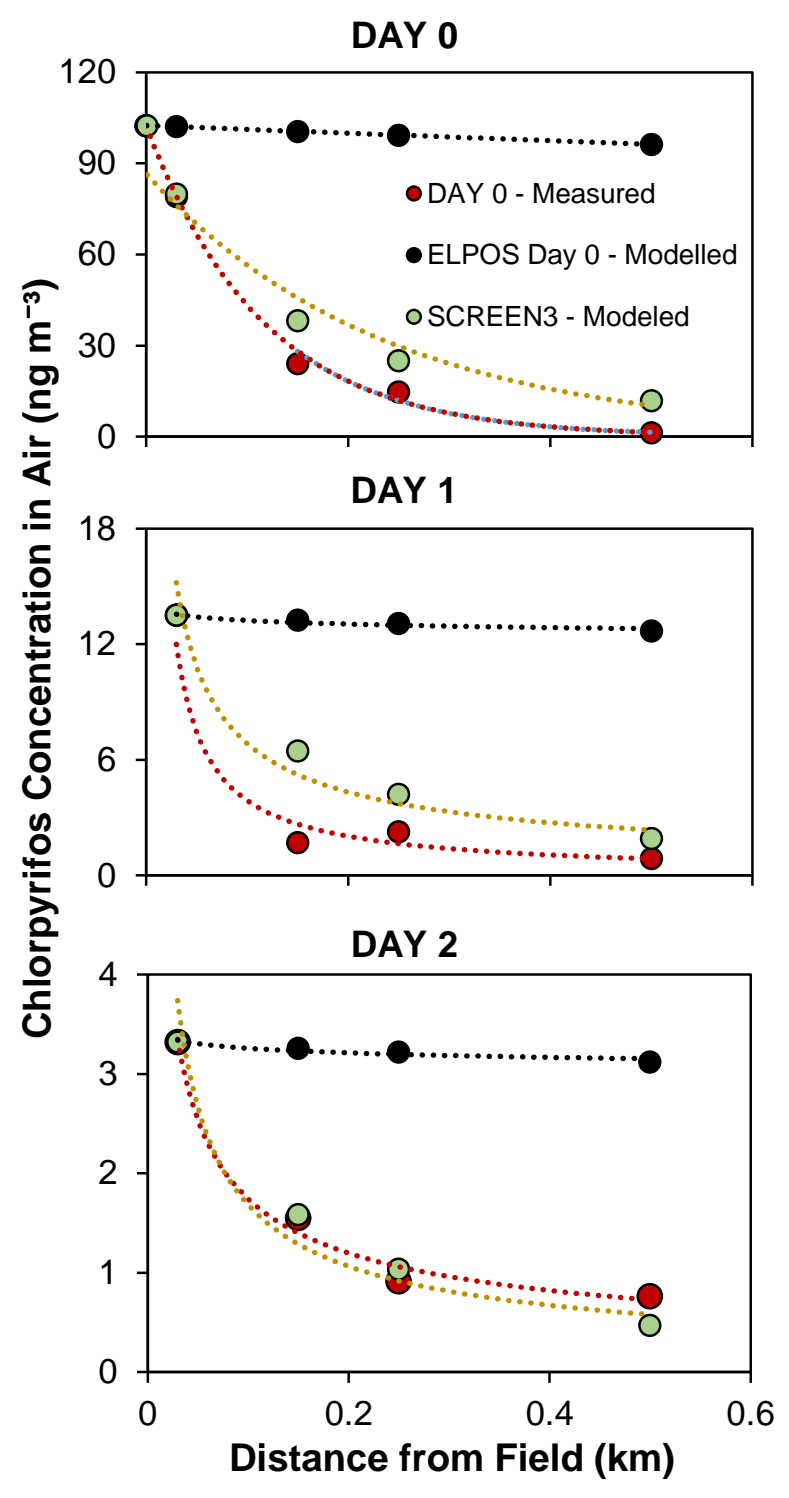

Figure 5. Measured, SCREEN3-modelled, and ELPOS-modelled chlorpyrifos concentrations on Days 0,1 , and 2 along the eastward-extending transect from our field site. Day 0 fitted lines are exponential while Day 1 and 2 fitted lines are power curves. Modeled concentrations were normalized to start at the same concentration as measured ones.

The CTD predicted by ELPOS ( $8 \mathrm{~km}$ ) was used in Equation 2 to obtain the ELPOS-predicted

406 concentrations shown in Figure 5. Our initial measured concentrations were used as $C_{0}$ in Equation

4072 so that the measured and modelled concentrations near the field edge were identical. The

408 concentration decrease predicted by the CTD was much lower than what we observed, indicating

409 that the change in chlorpyrifos concentration with distance from the field was not significantly 
410 affected by deposition processes, which ELPOS is designed to predict. In sum, these results

411 suggest that relatively simple air dispersion models like SCREEN3 should accurately predict

412 concentration loss trends with distance from source for semi-volatile pesticides. However,

413 SCREEN3 should be used with caution for pesticides that undergo more rapid degradation in the

414 atmosphere and/or when meteorology is more complex that it was in study.

\subsection{Comparison of chlorpyrifos concentrations in air to human health standards}

A handful of human health standards for chlorpyrifos concentrations in air have been

418 produced by US agencies; however, as indicated in the review by Li and Jennings 2017, such

419 values are not available for other countries. The Texas short- and long-term hourly average Effects

420 Screening Level concentrations for chlorpyrifos in air are 100 and $1000 \mathrm{ng} \mathrm{m}^{-3}$, respectively (Texas

421 Commission on Environmental Quality 2014). The US EPA uses reference concentrations ranging

422 from 2.1 to $51 \mathrm{ng} \mathrm{m}^{-3}$ of chlorpyrifos in air in its Revised Human Health Risk Assessment for

423 chlorpyrifos (U.S. Environmental Protection Agency 2016). The California EPA uses reference

424 concentrations ranging from 4.1 to $8.6 \mathrm{ng} \mathrm{m}^{-3}$ chlorpyrifos its Final Toxic Air Contaminant

425 Evaluation of Chlorpyrifos (California Environmental Protection Agency 2018). The reference

426 concentrations quoted here are the Critical Points of Departure, which were calculated with a

427 physiologically-based pharmacokinetic-pharmacodynamic (PBPK-PD) model (U.S.

428 Environmental Protection Agency 2006), reduced by a factor of 100. Thus, the chlorpyrifos

429 concentrations we measured in air on Days 0-2 up to $0.5 \mathrm{~km}$ from the field (Figure 5), and to some

430 extent beyond Day 2 (Table S12) at various locations, were within the range of concern for human

431 health according to the US and California EPA assessments. In their health assessments, the US

432 EPA compared reference concentrations to those measured in several field experiments whereas 
433 the California EPA compared reference concentrations to modelled concentrations generated with

434 the AGricultural DISPersal near-wake Lagrangian model (AGDISP) model using default

435 application scenarios. The use of measured and modelled data in these health assessments

436 highlights the importance of a thorough understanding of the factors that affect chlorpyrifos

437 concentrations in air in near- and downwind locations from sprayed fields.

\section{CONCLUSIONS}

440 Chlorpyrifos was detected in all in-field and near-field matrices (leaf, soil and air) at higher 441 than background concentrations until 21 days after application. Chlorpyrifos oxon was also 442 detected in near-field air samples with HVAS for the first three days after spraying. Chlorpyrifos 443 concentrations decreased rapidly in all matrices during the first several days after application. 444 Several observed concentration spikes could be explained by shifts in wind direction. The 445 concentrations measured on different sides of the field were remarkably varied and could generally 446 be explained by predominant wind directions. Measured chlorpyrifos trends generally agreed with 447 the SCREEN3 predicted trends. By contrast, ELPOS failed to predict those concentrations, 448 indicating that air dispersion was mainly responsible for the observed concentration trends along

449 the transect extending away from the field. The concentrations measured in air on Days 0-2, at 450 locations up to $0.5 \mathrm{~km}$ from the field, were at concentrations considered concerning for human 451 health.

452 Future research should focus on improving our understanding of the various field and 453 meteorological factors that affect pesticide $\mathrm{DT}_{50}$ values in leaves, soil, and air. This information 454 could be used to refine pesticide management decisions. For example, $\mathrm{DT}_{50}$ values on leaves and 455 soil control the length of time during which pesticides are effective against pests and harmful to 
managed bees and beneficial insects. In addition, this research shows that better methods are

457 needed to predict and understand the emission rates of semi-volatile pesticides from agricultural

458 fields since reliable values are needed as input parameters in air dispersion models such as

459 SCREEN3. Among other things, air dispersion models can be used to predict pesticide inhalation 460 exposure to farmworkers and bystanders.

\section{ACKNOWLEDGEMENTS}

The authors especially wish to thank Garth Tyrrell and John Wells for their help with 464 designing and building the medium-volume active air samplers. For logistical and field assistance, 465 we would also like to thank the Paterson family (Ida Valley Station) and Tim Duncan (Duncan 466 Agricultural Contractors, Poolburn), Matthew Hayward (Department of Zoology, University of 467 Otago), and Robert Alumbaugh (Department of Pharmacology \& Toxicology, University of 468 Otago). S. Das and S. Michelsen-Heath were sponsored by University of Otago PhD scholarships.

\section{SUPPLEMENTARY INFORMATION}

The supplementary information file contains additional details about methods, 472 meteorological conditions, and results, as well as tables of measured concentrations.

\section{REFERENCES} and mass spectrometric identification of chlorpyrifos and its two metabolites on field-grown collard and kale. Journal of Environmental Science and Health, Part B 0: 1-5. 
Bennett, D. H., McKone, T. E., Matthies, M. and Kastenberg, W. E. (1998). General Formulation of Characteristic Travel Distance for Semivolatile Organic Chemicals in a Multimedia Environment. Environmental Science \& Technology 32: 4023-4030.

Beyer, A. and Matthies, M. (2002). Criteria for atmospheric transport potential and persistence of pesticides and industrial chemicals. German Environmental Agency, Report No. 02/2002.

California Environmental Protection Agency. (2018). Final Toxic Air Contaminant Evaluation of Chlorpyrifos (https://www.cdpr.ca.gov/docs/whs/pdf/chlorpyrifos_final_tac.pdf) (Accessed October 2019).

California Environmental Protection Agency. (2019). California acts to prohibit chlorpyrifos pesticide (https://calepa.ca.gov/2019/05/08/california-acts-to-prohibit-chlorpyrifos-pesticide/) (Accessed June 2019).

Centner, T. J. (2018). Cancelling pesticide registrations and revoking tolerances: The case of chlorpyrifos. Envrionmental Toxicology and Pharmacology 57: 53-61.

European Food Safety Authority (2015). Reasoned opinion on the refined risk assessment regarding certain maximum residue levels (MRLs) of concern for the active substance chlorpyrifos. EFSA Journal 13: 4142.

Flaskos, J. (2012). The developmental neurotoxicity of organophosphorus insecticides: A direct role for the oxon metabolites. Toxicology Letters 209: 86-93.

Gao, J., Wang, Y., Goa, B., Wu, L. and Chen, H. (2012). Environmental Fate and Transport of Pesticides. Pesticides: Evaluation of Environmental Pollution. H. S. Rathore and L. M. L. Nollet. Boca Raton, FL, CRC Press.

Guardino, X., Obiols, J., Rosell, M. G., Farran, A. and Serra, C. (1998). Determination of chlorpyrifos in air, leaves and soil from a greenhouse by gas-chromatography with nitrogenphosphorus detection, high-performance liquid chromatography and capillary electrophoresis. Journal of Chromatography A 823: 91-96.

John, E. M. and Shaike, J. M. (2015). Chlorpyrifos: pollution and remediation. Environmental Chemistry Letters 13: 269-291.

Lakes

Environmental.

(2019).

SCREEN

View (https://www.weblakes.com/products/screen/index.html) (Accessed June 2019).

Leistra, M., Smelt, J. H., Hilbrand Weststrate, J., Van Den Berg, F. and Aalderink, R. (2006). Volatilization of the pesticides chlorpyrifos and fenpropimorph from a potato crop. Environmental Science and Technology 40: 96-102.

Lester, Y., Sabach, S., Zivan, O. and Dubowski, Y. (2017). Key environmental processes affecting the fate of the insecticide chlorpyrifos applied to leaves. Chemosphere 171: 74-80. 
Li, Z. and Jennings, A. (2017). Worldwide regulations of standard values of pesticides for human health risk control: A review. International Journal of Environmental Research and Public Health 14: 826 .

Mackay, D., Giesy, J. P. and Solomon, K. R. (2014). Fate in the environment and long-range Environmental Contamination and Toxicology. J. P. Giesy and K. R. Soloman. Heidelberg, Springer Open.

Mie, A., Rudén, C. and Grandjean, P. (2017). Safety of safety evaluation of pesticides: developmental neurotoxicity of chlorpyrifos and chlorpyrifos-methyl. Environmental Health 17: 77.

Montemurro, N., Grieco, F., Lacertosa, G. and Visconti, A. (2002). Chlorpyrifos decline curves and residue levels from different commercial formulations applied to oranges. Journal of Agricultural and Food Chemistry 50: 5975-5980.

Ngan, C. K., Cheah, U. B., Abdullah, W. Y. W., Lim, K. P. and Ismail, B. S. (2005). Fate of chlorothalonil, chlorpyrifos and profenofos in a vegetable farm in Cameron Highlands, Malaysia. Water, Air, and Soil Pollution: Focus 5: 125-136.

Perera, F. P., Rauh, V., Whyatt, R. M., Tang, D., Tsai, W. Y., Bernert, J. T., Tu, Y. H., Andrews, H., Barr, D. B., Camann, D. E., Diaz, D., Dietrich, J., Reyes, A. and Kinney, P. L. (2005). A summary of recent findings on birth outcomes and developmental effects of prenatal ETS, PAH, and pesticide exposures. Neurotoxicology 26: 573-587.

Pesticide Action Network International. (2019). Pan International Consolidated List of Banned Pesticides (http://pan-international.org/pan-international-consolidated-list-of-banned-pesticides/) (Accessed June 2019).

Racke, K. D. (1993). Environmental Fate of Chlorpyrifos. Reviews of Environmental Contamination and Toxicology. G. W. Ware. New York, Springer: 1-150.

Rauh, V., Arunajadai, S., Horton, M., Perera, F., Hoepner, L., Barr, D. B. and Whyatt, R. (2011). Seven-year neurodevelopmental scores and prenatal exposure to chlorpyrifos, a common agricultural pesticide. Environmental Health Perspectives 119: 1196-1201.

Rayment, G. E. and Lyons, D. J. (2011). Soil Chemical Methods: Australasia (CSIRO Publications).

Sanchez-Bayo, F. and Goka, K. (2014). Pesticide residues and bees - a risk assessment. Plos One 9: e94482.

Sarmah, A. K., Müller, K. and Ahmad, R. (2004). Fate and behaviour of pesticides in the acroecosystem - a review with a New Zealand perspective. Australian Journal of Soil Research 42: 125-154. 
Silver, M. K., Shao, J., Zhu, B., Chen, M., Xia, Y., Kaciroti, N., Lozoff, B. and Meeker, J. D. (2017). Prenatal naled and chlorpyrifos exposure is associated with deficits in infant motor function in a cohort of Chinese infants. Environment International 106: 248-256.

Solomon, K. R., Williams, W. M., Mackay, D., Purdy, J., Giddings, J. M. and Giesy, J. P. (2014). and Toxicology. 231: 13-34.

Testai, E., Buratti, F. M. and Di Consiglio, E. (2010). Chapter 70 - Chlorpyrifos. Hayes' Handbook of Pesticide Toxicology (Third Edition). R. Krieger. New York, Academic Press: 1505-1526.

Texas Commission on Environmental Quality. (2014). Effects Screening Levels (https://www.tceq.texas.gov/toxicology/esl/list_main.html) (Accessed October 2019).

Tomlin, C. D. S. (2006). The Pesticide Manual, A World Compendium, 14th Ed.; British Crop Protection Council. Alton, UK.

U.S. Environmental Protection Agency (2006). Approaches for the Application of Physiologically Based Pharmacokinetic (PBPK) Models and Supporting Data in Risk Assessment (https://ofmpub.epa.gov/eims/eimscomm.getfile?p_download_id=458188) (Accessed October 2019).

U.S. Environmental Protection Agency. (2011). Revised chlorpyrifos preliminary registration review drinking water assessment (http://www.epa.gov/oppsrrd1/registration_review/chlorpyrfios/EPA-HQ-OPP-2008-0850DRAFT-0025\%5B1\%5D.pdf (Accessed September 2019).

U.S. Environmental Protection Agency. (2016). Chlorpyrifos: Revised Human Health Risk Assessment for Registration Review (https://www.regulations.gov/document?D=EPA-HQ-OPP2015-0653-0454) (Accessed October 2019).

U.S. Environmental Protection Agency. (2018). Environmental transport and fate data analysis for chlorpyrifos, Appendix 3-1. (https://www3.epa.gov/pesticides/nas/final/chlorpyrifos/appendix-31.docx) (Accessed November 2018).

U.S. Environmental Protection Agency. (2019). Air Quality Dispersion Modeling - Screening Models (https://www.epa.gov/scram/air-quality-dispersion-modeling-screening-models\#screen3) (Accessed June 2019).

Woodrow, J. E., Seiber, J. N. and Baker, L. W. (1997). Correlation techniques for estimating pesticide volatilization flux and downwind concentrations. Environmental Science and Technology 31: 523-529.

Zivan, O., Segal-Rosenheimer, M. and Dubowski, Y. (2016). Airborne organophosphate pesticides drift in Mediterranean climate: The importance of secondary drift. Atmospheric Environment 127: 155-162. 